\title{
The Extended Trial Equation Method for Some Time Fractional Differential Equations
}

\author{
Yusuf Pandir, ${ }^{1}$ Yusuf Gurefe, ${ }^{1}$ and Emine Misirli ${ }^{2}$ \\ ${ }^{1}$ Department of Mathematics, Faculty of Science and Arts, Bozok University, 66100 Yozgat, Turkey \\ ${ }^{2}$ Department of Mathematics, Faculty of Science, Ege University, 35100 Izmir, Turkey \\ Correspondence should be addressed to Yusuf Gurefe; ygurefe@gmail.com
}

Received 21 February 2013; Revised 25 March 2013; Accepted 14 April 2013

Academic Editor: Ferhan M. Atici

Copyright (C) 2013 Yusuf Pandir et al. This is an open access article distributed under the Creative Commons Attribution License, which permits unrestricted use, distribution, and reproduction in any medium, provided the original work is properly cited.

\begin{abstract}
Nonlinear fractional partial differential equations have been solved with the help of the extended trial equation method. Based on the fractional derivative in the sense of modified Riemann-Liouville derivative and traveling wave transformation, the fractional partial differential equation can be turned into the nonlinear nonfractional ordinary differential equation. For illustrating the reliability of this approach, we apply it to the generalized third order fractional KdV equation and the fractional $K(n, n)$ equation according to the complete discrimination system for polynomial method. As a result, some new exact solutions to these nonlinear problems are successfully constructed such as elliptic integral function solutions, Jacobi elliptic function solutions, and soliton solutions.
\end{abstract}

\section{Introduction}

Many physical phenomena in mathematical biology, electromagnetic theory, fluid mechanics, signal processing, engineering, and other fields of science can be modeled using the fractional derivatives. For example, the use of fractional derivative is analyzed in the fields of controller tuning, redundant robots, legged robots, digital circuit synthesis, and heat diffusion [1]. Also, we refer the reader to $[1,2]$ for details concerning fractional derivative or calculus and their applications in science and engineering. In some sense fractional differential equations could represent various reallife problems [3-5]. However, an effective and general method for solving them cannot be found even in the most useful works. Also, remarkable progress has been made in the construction of the approximate solutions for fractional nonlinear partial differential equations. There are various mathematical methods which can constitute the exact solutions for some time fractional differential equations $[6,7]$. Single kink soliton solutions, multiple-soliton solutions, compacton-like solutions, singular solitons, and other solutions have been found by use of these approaches. Apart from all these, some new exact solutions are obtained by using the trial equation methods. Some of them are elliptic integral $F, E$, and $\Pi$ functions, and Jacobi elliptic function solutions.

In Section 2, primarily we give some definitions and properties of the fractional calculus theory and also produce the extended trial equation method for fractional nonlinear evolution equations with higher order nonlinearity. The power of this steerable method showed that this method can be applied to two nonlinear fractional problems. In Section 3, as applications, we solve the fractional nonlinear partial differential equations such as the generalized third order fractional $\mathrm{KdV}$ equation [8]

$$
\frac{\partial^{\alpha} u}{\partial t^{\alpha}}+u_{x x x}+u^{p} u_{x}=0, \quad-\infty<x<\infty, t>0,
$$

where $0<\alpha \leq 1$ and $p \geq 2$ model the subdiffusion and the subdispersion term, and the fractional $K(n, n)$ equation [9]

$$
\begin{aligned}
& \frac{\partial^{\alpha} u}{\partial t^{\alpha}}+\left(u^{n}\right)_{x}+\left(u^{n}\right)_{x x x} \\
& =0, \quad-\infty<x<\infty, t>0,0<\alpha \leq 1,
\end{aligned}
$$

where $n>1$. Equation (1) has a wide range of applications in plasma physics, fluid physics, and capillary-gravity waves. 
Although there are a lot of studies for the classical generalized third order $\mathrm{KdV}$ and the $K(n, n)$ equation, this is the first study with respect to the fractional versions of these problems. The aim of this paper is to obtain exact solutions of the generalized third order fractional $\mathrm{KdV}$ and the fractional $K(n, n)$ equation by extendend trial equation method and to determine the accuracy of extended trial equation method in solving these kinds of problems. The exact solutions obtained by the interesting wave transformation $\eta=k x-$ $\left(\lambda t^{\alpha} / \Gamma(1+\alpha)\right)$ are used to determine some new phenomena of the fractional nonlinear evolution equations. Based on the derived solutions for (1) and (2), the phenomena of some solutions are shown graphically (see Figures 1, 2, and 3).

\section{Preliminaries and the Extended Trial Equation Method}

In this part of the paper, it would be helpful to give some definitions and properties of the fractional calculus theory. For an introduction to the classical fractional calculus we indicate the reader to [3-5]. Here, we shortly review the modified Riemann-Liouville derivative from the recent fractional calculus proposed by Jumarie [10-12]. Let $f:[0,1] \rightarrow \mathbb{R}$ be a continuous function and $\alpha \in(0,1)$. The Jumarie modified fractional derivative of order $\alpha$ and $f$ may be defined by expression of [13] as follows:

$$
\begin{aligned}
& D_{x}^{\alpha} f(x) \\
& =\left\{\begin{array}{l}
\frac{1}{\Gamma(-\alpha)} \int_{0}^{x}(x-\xi)^{-\alpha-1}[f(\xi)-f(0)] d \xi, \\
\alpha<0, \\
\frac{1}{\Gamma(1-\alpha)} \frac{d}{d x} \int_{0}^{x}(x-\xi)^{-\alpha}[f(\xi)-f(0)] d \xi, \\
0<\alpha<1, \\
\left(f^{(n)}(\xi)\right)^{\alpha-n}, \quad n \leq \alpha \leq n+1, n \geq 1 .
\end{array}\right.
\end{aligned}
$$

In addition to this expression, we may give a summary of the fractional modified Riemann-Liouville derivative properties which are used further in this paper. Some of the useful formulas are given as

$$
D_{x}^{\alpha} x^{\mu}= \begin{cases}0, & \mu \leq \alpha-1, \\ \frac{\Gamma(\mu+1)}{\Gamma(\mu-\alpha+1)} x^{\mu-\alpha}, & \mu>\alpha-1 .\end{cases}
$$

Some new trial equation methods were defined in the literature [14-26]. In this paper, the extended trial equation method will be given. In order to apply this method to fractional nonlinear partial differential equations, we consider the following steps.

Step 1. Consider time fractional partial differential equation in two variables and a dependent variable $u$

$$
P\left(u, D_{t}^{\alpha} u, u_{x}, u_{x x}, u_{x x x}, \ldots\right)=0
$$

and take the wave transformation

$$
u(x, t)=u(\eta), \quad \eta=k x-\frac{\lambda t^{\alpha}}{\Gamma(1+\alpha)}
$$

where $\lambda \neq 0$. Substituting (6) into (5) yields a nonlinear ordinary differential equation

$$
N\left(u, u^{\prime}, u^{\prime \prime}, \ldots\right)=0
$$

Step 2. Take trial equation as follows:

$$
u=\sum_{i=0}^{\delta} \tau_{i} \Upsilon^{i}
$$

where

$$
\left(\Upsilon^{\prime}\right)^{2}=\Lambda(\Upsilon)=\frac{\Phi(\Upsilon)}{\Psi(\Upsilon)}=\frac{\xi_{\theta} \Upsilon^{\theta}+\cdots+\xi_{1} \Upsilon+\xi_{0}}{\zeta_{\epsilon} \Upsilon^{\epsilon}+\cdots+\zeta_{1} \Upsilon+\zeta_{0}}
$$

Using (8) and (9), we can write

$$
\begin{gathered}
\left(u^{\prime}\right)^{2}=\frac{\Phi(\Upsilon)}{\Psi(\Upsilon)}\left(\sum_{i=0}^{\delta} i \tau_{i} \Upsilon^{i-1}\right)^{2}, \\
u^{\prime \prime}=\frac{\Phi^{\prime}(\Upsilon) \Psi(\Upsilon)-\Phi(\Upsilon) \Psi^{\prime}(\Upsilon)}{2 \Psi^{2}(\Upsilon)}\left(\sum_{i=0}^{\delta} i \tau_{i} \Upsilon^{i-1}\right) \\
+\frac{\Phi(\Upsilon)}{\Psi(\Upsilon)}\left(\sum_{i=0}^{\delta} i(i-1) \tau_{i} \Upsilon^{i-2}\right)
\end{gathered}
$$

where $\Phi(\Upsilon)$ and $\Psi(\Upsilon)$ are polynomials. Substituting the above relations into (7) yields an equation of polynomial $\Omega(\Upsilon)$ of $\Upsilon$ :

$$
\Omega(\Upsilon)=\varrho_{s} \Upsilon^{s}+\cdots+\varrho_{1} \Upsilon+\varrho_{0}=0
$$

According to the balance principle, we can find a relation of $\theta, \epsilon$, and $\delta$. We can compute some values of $\theta, \epsilon$, and $\delta$.

Step 3. Let the coefficients of $\Omega(\Upsilon)$ all be zero will yield an algebraic equations system:

$$
\varrho_{i}=0, \quad i=0, \ldots, s .
$$

Solving this system, we will determine the values of $\xi_{0}, \ldots, \xi_{\theta}$, $\zeta_{0}, \ldots, \zeta_{\epsilon}$ and $\tau_{0}, \ldots, \tau_{\delta}$.

Step 4. Reduce (9) to the elementary integral form

$$
\pm\left(\eta-\eta_{0}\right)=\int \frac{d \Upsilon}{\sqrt{\Lambda(\Upsilon)}}=\int \sqrt{\frac{\Psi(\Upsilon)}{\Phi(\Upsilon)}} d \Upsilon
$$

Using a complete discrimination system for polynomial to classify the roots of $\Phi(\Upsilon)$, we solve (13) with the help of Mathematica and classify the exact solutions to (7). In addition, we can write the exact traveling wave solutions to (5), respectively. 


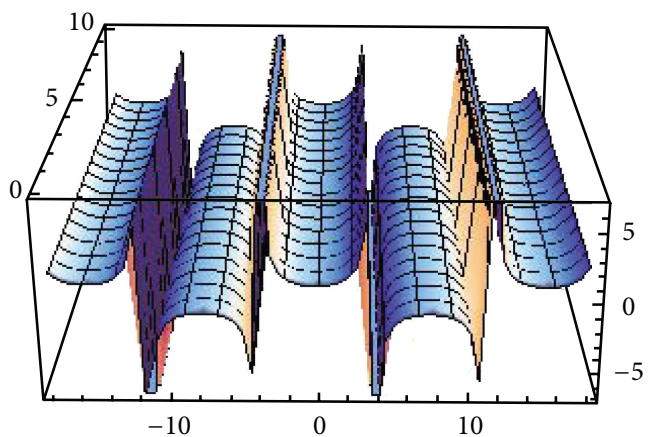

(a)

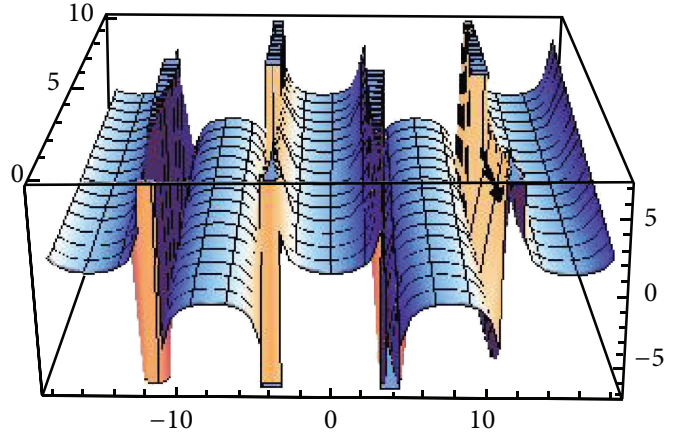

(b)

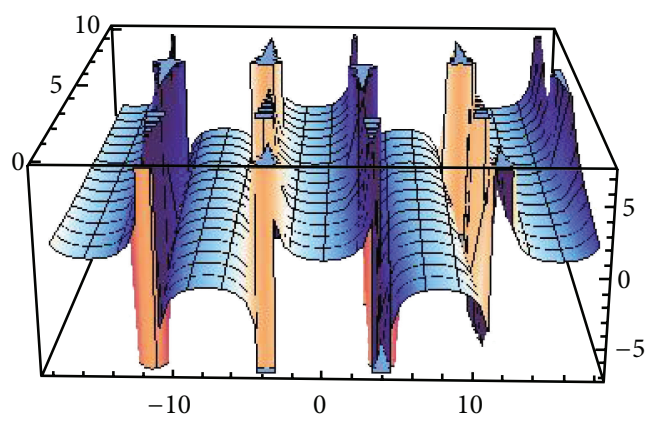

(c)

FIGURE 1: Graph of the solution (31) corresponding to the values $\alpha=0.01,0.50$ and $\alpha=0.85$ from (a) to (c) when $\tau_{1}=\alpha_{1}=\xi_{0}=\xi_{1}=k=1$, $p=\alpha_{1}=2$.

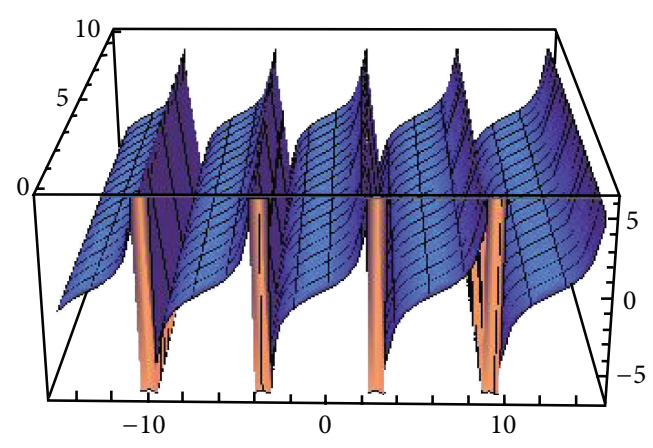

(a)

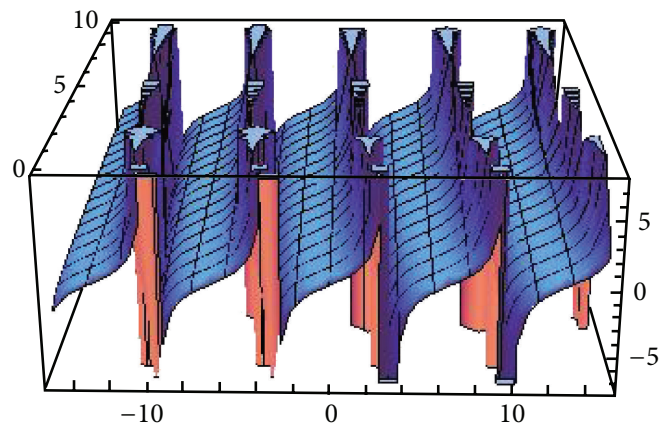

(b)

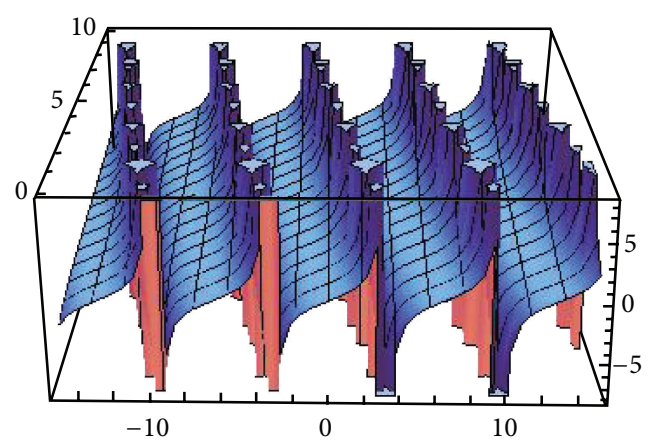

(c)

Figure 2: Graph of the solution (33) corresponding to the values $\alpha=0.01,0.50$ and $\alpha=0.85$ from (a) to (c) when $\tau_{1}=\alpha_{3}=\xi_{0}=\xi_{1}=k=1$, $p=\alpha_{2}=2$, and $\alpha_{1}=3$. 


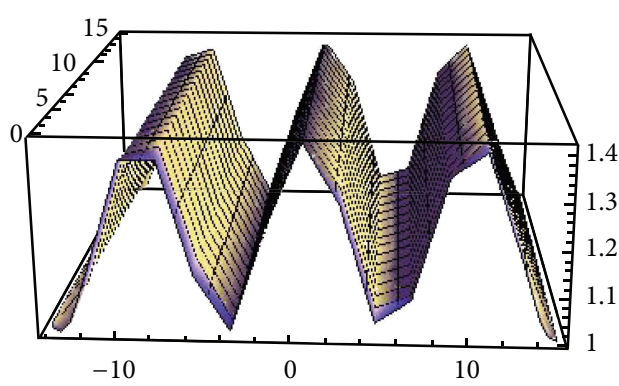

(a)

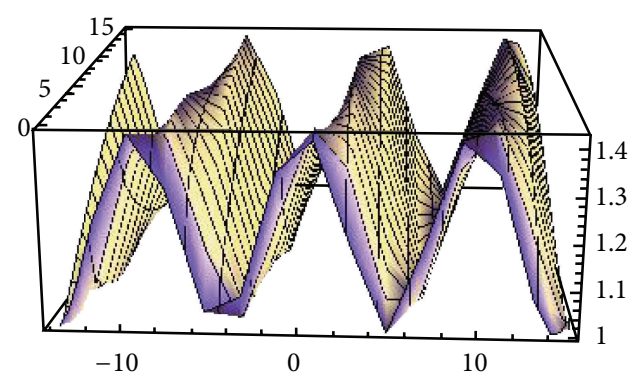

(b)

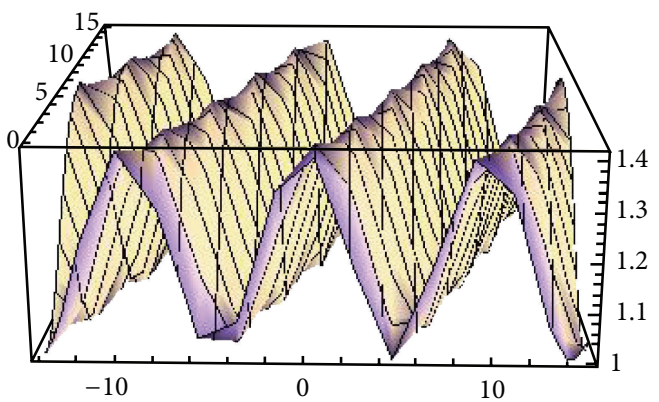

(c)

Figure 3: Graph of the solution (60) corresponding to the values $\alpha=0.01,0.50$ and $\alpha=0.85$ from (a) to (c) when $\tau_{0}=\tau_{1}=\xi_{1}=k=1$, $n=3$, and $\xi_{2}=3 / 2$.

\section{Applications to the Extended Trial Equation Method}

In this section, we apply the method given in Section 2 to the generalized third order fractional $\mathrm{KdV}$ and the fractional $K(n, n)$ equation, respectively.

3.1. Application to Generalized Fractional KdV Equation. In the case of $\alpha=1$, (1) reduces to the classical nonlinear generalized third order KdV equation. Many researchers have tried to get the exact solutions of this equation by using a variety of methods. But, we solve the generalized fractional $\mathrm{KdV}$ equation for $0<\alpha \leq 1$. Compactons, solitary patterns, and periodic and solitary traveling plane waves solutions of this equation are found.

Let us consider the travelling wave solutions of (1), and we perform the transformation $u(x, t)=u(\eta), \eta=k x-$ $\left(\lambda t^{\alpha} / \Gamma(1+\alpha)\right)$, where $\lambda$ is constant to be determined later. Then, integrating this equation with respect to $\eta$ and setting the integration constant to zero, we get

$$
-\lambda(p+1) u+k^{3}(p+1) u^{\prime \prime}+k u^{p+1}=0 .
$$

By use of the following transformation:

$$
u=v^{1 / p}
$$

(14) turns into the equation

$$
\begin{array}{r}
k^{3}\left(p^{2}+p\right) v v^{\prime \prime}+k^{3}\left(1-p^{2}\right)\left(v^{\prime}\right)^{2} \\
-\lambda\left(p^{3}+p^{2}\right) v^{2}+k p^{2} v^{3}=0 .
\end{array}
$$

From (8)-(10), we can write the following highest order nonlinear terms in order to determine the balance procedure:

$$
\begin{gathered}
v=\tau_{\delta} \Upsilon^{\delta}+\cdots \\
v^{3}=\tau_{\delta}^{3} \Upsilon^{3 \delta}+\cdots \\
\left(v^{\prime}\right)^{2}=\frac{\xi_{\theta} \delta^{2} \tau_{\delta}^{2}}{\zeta_{\epsilon}} \Upsilon^{2 \delta-2}\left(\Upsilon^{\prime}\right)^{2}+\cdots=\frac{\xi_{\theta} \delta^{2} \tau_{\delta}^{2}}{\zeta_{\epsilon}} \Upsilon^{2 \delta+\theta-\epsilon-2}+\cdots, \\
v v^{\prime \prime}=\omega \Upsilon^{2 \delta+\theta-\epsilon-2}+\cdots .
\end{gathered}
$$

Balancing the highest order nonlinear terms of $v v^{\prime \prime}$ and $v^{3}$ in (16), we have

$$
2 \delta+\theta-\epsilon-2=3 \delta,
$$

hence

$$
\theta=\epsilon+\delta+2
$$

This resolution procedure is applied, and we obtain results as follows.

Case 1. If we take $\epsilon=0, \delta=1$, and $\theta=3$, then

$$
\begin{aligned}
\left(v^{\prime}\right)^{2} & =\frac{\left(\tau_{1}\right)^{2}\left(\xi_{3} \Upsilon^{3}+\xi_{2} \Upsilon^{2}+\xi_{1} \Upsilon+\xi_{0}\right)}{\zeta_{0}}, \\
v^{\prime \prime} & =\frac{\left(\tau_{1}\right)\left(3 \xi_{3} \Upsilon^{2}+2 \xi_{2} \Upsilon+\xi_{1}\right)}{2 \zeta_{0}},
\end{aligned}
$$


where $\xi_{3} \neq 0, \zeta_{0} \neq 0$. Thus, we have a system of algebraic equations from the coefficients of polynomial of $\Upsilon$. Solving the algebraic equation system (12), we get

$$
\begin{gathered}
\xi_{0}=\xi_{0}, \quad \xi_{1}=\xi_{1}, \\
\xi_{2}=\frac{\tau_{1}\left(2 \xi_{1} \tau_{0}-3 \xi_{0} \tau_{1}\right)}{\tau_{0}^{2}}, \quad \xi_{3}=\frac{\tau_{1}^{2}\left(\xi_{1} \tau_{0}-2 \xi_{0} \tau_{1}\right)}{\tau_{0}^{3}}, \\
\tau_{0}=\tau_{0}, \quad \tau_{1}=\tau_{1}, \\
\zeta_{0}=-\frac{k^{2}(1+p)(2+p) \tau_{1}\left(\xi_{1} \tau_{0}-2 \xi_{0} \tau_{1}\right)}{2 p^{2} \tau_{0}^{3}}, \\
\lambda=\frac{2 k \tau_{0}\left(\xi_{1} \tau_{0}-3 \xi_{0} \tau_{1}\right)}{\left(2+3 p+p^{2}\right)\left(\xi_{1} \tau_{0}-2 \xi_{0} \tau_{1}\right)} .
\end{gathered}
$$

Substituting these results into (13), we have

$$
\begin{aligned}
& \pm\left(\eta-\eta_{0}\right) \\
& =\sqrt{-\frac{k^{2}\left(2+3 p+p^{2}\right)}{2 p^{2} \tau_{1}}} \\
& \quad \times \int\left(d \Upsilon \times\left(\Upsilon^{3}+\frac{\tau_{0}\left(2 \xi_{1} \tau_{0}-3 \xi_{0} \tau_{1}\right)}{\tau_{1}\left(\xi_{1} \tau_{0}-2 \xi_{0} \tau_{1}\right)} \Upsilon^{2}\right.\right. \\
& +\frac{\xi_{1} \tau_{0}^{3}}{\tau_{1}^{2}\left(\xi_{1} \tau_{0}-2 \xi_{0} \tau_{1}\right)} \Upsilon \\
& \left.\left.+\frac{\xi_{0} \tau_{0}^{3}}{\tau_{1}^{2}\left(\xi_{1} \tau_{0}-2 \xi_{0} \tau_{1}\right)}\right)^{-1 / 2}\right) .
\end{aligned}
$$

Integrating (22), we procure the solutions to (1) as follows:

$$
\begin{gathered}
\pm\left(\eta-\eta_{0}\right)=-\frac{2 A}{\sqrt{\Upsilon-\alpha_{1}}}, \\
\pm\left(\eta-\eta_{0}\right)=\frac{2 A}{\sqrt{\alpha_{2}-\alpha_{1}}} \arctan \sqrt{\frac{\Upsilon-\alpha_{2}}{\alpha_{2}-\alpha_{1}}}, \quad \alpha_{2}>\alpha_{1}, \\
\pm\left(\eta-\eta_{0}\right)=\frac{A}{\sqrt{\alpha_{1}-\alpha_{2}}} \ln \mid \frac{\sqrt{\Upsilon-\alpha_{2}}-\sqrt{\alpha_{1}-\alpha_{2}}}{\sqrt{\Upsilon-\alpha_{2}}+\sqrt{\alpha_{1}-\alpha_{2}}}, \quad \alpha_{1}>\alpha_{2}, \\
\pm\left(\eta-\eta_{0}\right)=-\frac{2 A}{\sqrt{\alpha_{1}-\alpha_{3}}} F(\varphi, l), \quad \alpha_{1}>\alpha_{2}>\alpha_{3},
\end{gathered}
$$

where

$$
\begin{aligned}
& A=\sqrt{-\frac{k^{2}\left(2+3 p+p^{2}\right)}{2 p^{2} \tau_{1}}}, \quad F(\varphi, l)=\int_{0}^{\varphi} \frac{d \psi}{\sqrt{1-l^{2} \sin ^{2} \psi}}, \\
& \varphi=\arcsin \sqrt{\frac{\Upsilon-\alpha_{3}}{\alpha_{2}-\alpha_{3}}}, \quad l^{2}=\frac{\alpha_{2}-\alpha_{3}}{\alpha_{1}-\alpha_{3}} .
\end{aligned}
$$

Also $\alpha_{1}, \alpha_{2}$, and $\alpha_{3}$ are the roots of the polynomial equation

$$
\Upsilon^{3}+\frac{\xi_{2}}{\xi_{3}} \Upsilon^{2}+\frac{\xi_{1}}{\xi_{3}} \Upsilon+\frac{\xi_{0}}{\xi_{3}}=0
$$

Substituting the solutions (23) into (8) and (15), we have

$$
\begin{gathered}
u(x, t)=\left[\tau_{0}+\tau_{1} \alpha_{1}+\frac{4 A^{2} \tau_{1}}{\left(k x-\left(2 k \tau_{0}\left(\xi_{1} \tau_{0}-3 \xi_{0} \tau_{1}\right) /\left(2+3 p+p^{2}\right)\left(\xi_{1} \tau_{0}-2 \xi_{0} \tau_{1}\right) \Gamma(1+\alpha)\right) t^{\alpha}-\eta_{0}\right)^{2}}\right]^{1 / p}, \\
u(x, t)=\left[\tau_{0}+\tau_{1} \alpha_{1}+\tau_{1}\left(\alpha_{2}-\alpha_{1}\right) \operatorname{sech}^{2}\left(\frac{\sqrt{\alpha_{1}-\alpha_{2}}}{2 A}\left(k x-\frac{2 k \tau_{0}\left(\xi_{1} \tau_{0}-3 \xi_{0} \tau_{1}\right)}{\left(2+3 p+p^{2}\right)\left(\xi_{1} \tau_{0}-2 \xi_{0} \tau_{1}\right) \Gamma(1+\alpha)} t^{\alpha}-\eta_{0}\right)\right)\right]^{1 / p}, \\
u(x, t)=\left[\tau_{0}+\tau_{1} \alpha_{1}+\tau_{1}\left(\alpha_{1}-\alpha_{2}\right) \operatorname{cosech}^{2}\left(\frac{\sqrt{\alpha_{1}-\alpha_{2}}}{2 A}\left(k x-\frac{2 k \tau_{0}\left(\xi_{1} \tau_{0}-3 \xi_{0} \tau_{1}\right)}{\left(2+3 p+p^{2}\right)\left(\xi_{1} \tau_{0}-2 \xi_{0} \tau_{1}\right) \Gamma(1+\alpha)} t^{\alpha}-\eta_{0}\right)\right)\right]^{1 / p}, \\
\begin{array}{c}
u(x, t)=\left[\tau_{0}+\tau_{1} \alpha_{3}+\right. \\
\left.\left.\quad\left(\operatorname{sn}^{2}\left( \pm \frac{\left(\sqrt{\alpha_{1}-\alpha_{3}}\right)}{2 A}\left(k x-\frac{\left.2 k \tau_{0}\left(\alpha_{1}\right)\right)}{\left(2+3 p+p^{2}\right)\left(\xi_{1} \tau_{0}-2 \xi_{0} \tau_{1}\right) \Gamma(1+\alpha)} t^{\alpha}-\eta_{0}\right), \frac{\left(\alpha_{2}-\alpha_{3}\right)}{\left(\alpha_{1}-\alpha_{3}\right)}\right)\right)^{-1}\right)\right]^{1 / p} .
\end{array}
\end{gathered}
$$


If we take $\tau_{0}=-\tau_{1} \alpha_{1}$ and $\eta_{0}=0$, then the solutions (26)-(28) can reduce to rational function solution

$$
u(x, t)=\left(\frac{\widetilde{A}}{x-\lambda_{1} t^{\alpha}}\right)^{2 / p}
$$

1-soliton wave solution

$$
u(x, t)=\frac{\widetilde{B}}{\cosh ^{2 / p}\left(B\left(x-\lambda_{1} t^{\alpha}\right)\right)},
$$

singular soliton solution

$$
u(x, t)=\frac{\widetilde{C}}{\sinh ^{2 / p}\left(C\left(x-\lambda_{1} t^{\alpha}\right)\right)},
$$

where $\widetilde{A}=2 A \sqrt{\tau_{1}} / k, \widetilde{B}=\left(\tau_{1}\left(\alpha_{2}-\alpha_{1}\right)\right)^{1 / p}, B=k \sqrt{\alpha_{1}-\alpha_{2}} /$ $2 A, \widetilde{C}=\left(\tau_{1}\left(\alpha_{1}-\alpha_{2}\right)\right)^{1 / p}, C=k \sqrt{\alpha_{1}-\alpha_{2}} / 2 A$, and $\lambda_{1}=$ $-\left(2 \tau_{1} \alpha_{1}\left(\xi_{1} \alpha_{1}+3 \xi_{0}\right) /\left(2+3 p+p^{2}\right)\left(\xi_{1} \alpha_{1}+2 \xi_{0}\right) \Gamma(1+\alpha)\right)$. Here, $\widetilde{B}$ and $\widetilde{C}$ are the amplitude of the soliton, while $B$ and $C$ are the inverse width of the solitons and $c$ is the velocity.

On the other hand, if we take $\tau_{0}=-\tau_{1} \alpha_{3}$ and $\eta_{0}=0$, then the Jacobi elliptic function solution (3.1) can be written in the form

$$
u(x, t)=A_{1} \operatorname{sn}^{2 / p}\left(B_{i}\left(x-\lambda_{1} t^{\alpha}\right), \frac{\alpha_{2}-\alpha_{3}}{\alpha_{1}-\alpha_{3}}\right),
$$

where $A_{1}=\left(\tau_{1}\left(\alpha_{2}-\alpha_{3}\right)\right)^{1 / p}$ and $B_{i}=(-1)^{i} k \sqrt{\alpha_{1}-\alpha_{3}} / 2 A$, $i=(1,2)$. Thus, we can say that the solitons exist for $\tau_{1}>0$.

Remark 1 . We provide a proof by verifying the exact solution (31) by direct substitution. If we take $\alpha=1$ and $p=2$, then (1) becomes

$$
u_{t}+u_{x x x}+u^{2} u_{x}=0
$$

where $\widetilde{B}=\sqrt{\tau_{1}\left(\alpha_{2}-\alpha_{1}\right)}, B=k \sqrt{\alpha_{1}-\alpha_{2}} / 2 A, A=$ $k \sqrt{-\left(3 / 2 \tau_{1}\right)}$, and $\lambda_{1}=-\left(\tau_{1} \alpha_{1}\left(\xi_{1} \alpha_{1}+3 \xi_{0}\right) / 6\left(\xi_{1} \alpha_{1}+2 \xi_{0}\right)\right)$. Here, $B=\sqrt{\tau_{1}\left(\alpha_{2}-\alpha_{1}\right) / 6}$.

Equation (31) is

$$
u(x, t)=\widetilde{B} \operatorname{sech}\left[B\left(x-\lambda_{1} t\right)\right] .
$$

If $\phi=x-\lambda_{1} t$, then

$$
\begin{gathered}
u(\phi)=\widetilde{B} \operatorname{sech}(B \phi), \\
u_{t}=\widetilde{B} B \lambda_{1} \tanh (B \phi) \operatorname{sech}(B \phi), \\
u_{x}=-\widetilde{B} B \tanh (B \phi) \operatorname{sech}(B \phi), \\
u^{2} u_{x}=-\widetilde{B}^{3} B \tanh (B \phi) \operatorname{sech}^{3}(B \phi), \\
u_{x x x}=\widetilde{B} B^{3} \tanh (B \phi)\left(6 \operatorname{sech}^{3}(B \phi)-\operatorname{sech}(B \phi)\right) .
\end{gathered}
$$

Substituting the above relations into the partial differential equation, we obtain the following equation:

$$
\begin{gathered}
\widetilde{B} B \tanh (B \phi) \operatorname{sech}(B \phi)\left[\lambda_{1}-B^{2}+\left(6 B^{2}-\widetilde{B}^{2}\right) \operatorname{sech}^{2}(B \phi)\right] \\
=-\frac{\tau_{1}\left(\xi_{1} \alpha_{1} \alpha_{2}+2 \xi_{0} \alpha_{2}+\xi_{0} \alpha_{1}\right)}{6\left(\xi_{1} \alpha_{1}+2 \xi_{0}\right)}=0 .
\end{gathered}
$$

Otherwise, we can write

$$
-\alpha_{1}^{2} \alpha_{2}=\frac{\xi_{0} \tau_{0}^{3}}{\tau_{1}^{2}\left(\xi_{1} \tau_{0}-2 \xi_{0} \tau_{1}\right)},
$$

where $\tau_{0}=-\tau_{1} \alpha_{1}$ and $\left(\Upsilon-\alpha_{1}\right)^{2}\left(\Upsilon-\alpha_{2}\right)=\Upsilon^{3}+\left(\xi_{2} / \xi_{3}\right) \Upsilon^{2}+$ $\left(\xi_{1} / \xi_{3}\right) \Upsilon+\left(\xi_{0} / \xi_{3}\right)=0$. From this equation, we find $\xi_{1} \alpha_{1} \alpha_{2}+$ $2 \xi_{0} \alpha_{2}+\xi_{0} \alpha_{1}=0$. Substituting this relation into the above equation yields $0=0$. Thus, it is seen that the obtained solution (31) is an exact solution of the fractional $\mathrm{KdV}$ equation.

Remark 2. The solutions (30)-(33) obtained by using the extended trial equation method for (1) have been checked by Mathematica. To our knowledge, rational function solution, 1-soliton solution, the singular soliton solution, and the Jacobi elliptic function that we find in this paper are not shown in the previous literature. These results are new traveling wave solutions of (1).

Case 2. If we take $\epsilon=0, \delta=2$, and $\theta=4$, then

$$
\begin{aligned}
\left(v^{\prime}\right)^{2}= & \frac{\left(\tau_{1}+2 \tau_{2} \Upsilon\right)^{2}\left(\xi_{4} \Upsilon^{4}+\xi_{3} \Upsilon^{3}+\xi_{2} \Upsilon^{2}+\xi_{1} \Upsilon+\xi_{0}\right)}{\zeta_{0}}, \\
v^{\prime \prime}= & \left(\left(\tau_{1}+2 \tau_{1} \Upsilon\right)\left(4 \xi_{4} \Upsilon^{3}+3 \xi_{3} \Upsilon^{2}+2 \xi_{2} \Upsilon+\xi_{1}\right)\right. \\
& \left.\quad+4 \tau_{2}\left(\xi_{4} \Upsilon^{4}+\xi_{3} \Upsilon^{3}+\xi_{2} \Upsilon^{2}+\xi_{1} \Upsilon+\xi_{0}\right)\right) \\
& \times\left(2 \zeta_{0}\right)^{-1},
\end{aligned}
$$

where $\xi_{4} \neq 0, \zeta_{0} \neq 0$. Respectively, solving the algebraic equation system (12) yields as follows:

$$
\begin{gathered}
\xi_{0}=\frac{\left(\xi_{4} \tau_{1}^{2}-\xi_{2} \tau_{1}^{2}\right)^{2}}{4 \xi_{4} \tau_{2}^{4}}, \quad \xi_{1}=\frac{\xi_{2} \tau_{1} \tau_{2}^{2}-\xi_{4} \tau_{1}^{3}}{\tau_{2}^{3}}, \\
\xi_{3}=\frac{2 \xi_{4} \tau_{1}}{\tau_{2}}, \quad \xi_{2}=\xi_{2}, \quad \xi_{4}=\xi_{4}, \\
\tau_{0}=\frac{\xi_{2} \tau_{2}^{2}-\xi_{4} \tau_{1}^{2}}{2 \xi_{4} \tau_{2}}, \quad \tau_{1}=\tau_{1}, \quad \tau_{2}=\tau_{2}, \\
\zeta_{0}=-\frac{2 k^{2}\left(2+3 p+p^{2}\right) \xi_{4}}{p^{2} \tau_{2}} \\
\lambda=-\frac{k\left(3 \xi_{4} \tau_{1}^{2}-2 \xi_{2} \tau_{2}^{2}\right)}{2\left(2+3 p+p^{2}\right) \xi_{4} \tau_{2}} .
\end{gathered}
$$


Substituting these results into (13), we get

$$
\begin{aligned}
& \pm\left(\eta-\eta_{0}\right) \\
& =\sqrt{-\frac{2 k^{2}\left(2+3 p+p^{2}\right)}{p^{2} \tau_{2}}} \\
& \times \int\left(d \Upsilon \left(\Upsilon^{4}+\frac{2 \tau_{1}}{\tau_{2}} \Upsilon^{3}+\frac{\xi_{2}}{\xi_{4}} \Upsilon^{2}\right.\right. \\
& +\frac{\xi_{2} \tau_{1} \tau_{2}^{2}-\xi_{4} \tau_{1}^{3}}{\tau_{2}^{3} \xi_{4}} \Upsilon{ }^{2} \\
& \left.\left.+\frac{\left(\xi_{4} \tau_{1}^{2}-\xi_{2} \tau_{1}^{2}\right)^{2}}{4 \xi_{4}^{2} \tau_{2}^{4}}\right)^{-1 / 2}\right) .
\end{aligned}
$$

Integrating (41), we obtain the solutions to (1) as follows:

$$
\begin{gathered}
\pm\left(\eta-\eta_{0}\right)=-\frac{A_{2}}{\Upsilon-\alpha_{1}}, \\
\pm\left(\eta-\eta_{0}\right)=\frac{2 A_{2}}{\alpha_{1}-\alpha_{2}} \sqrt{\frac{\Upsilon-\alpha_{2}}{\Upsilon-\alpha_{1}}}, \quad \alpha_{1}>\alpha_{2}, \\
\pm\left(\eta-\eta_{0}\right)=\frac{A_{2}}{\alpha_{1}-\alpha_{2}} \ln \left|\frac{\Upsilon-\alpha_{1}}{\Upsilon-\alpha_{2}}\right|,
\end{gathered}
$$

$$
\begin{aligned}
& \pm\left(\eta-\eta_{0}\right) \\
& =\frac{2 A_{2}}{\sqrt{\left(\alpha_{1}-\alpha_{2}\right)\left(\alpha_{1}-\alpha_{3}\right)}} \\
& \quad \times \ln \left|\frac{\sqrt{\left(\Upsilon-\alpha_{2}\right)\left(\alpha_{1}-\alpha_{3}\right)}-\sqrt{\left(\Upsilon-\alpha_{3}\right)\left(\alpha_{1}-\alpha_{2}\right)}}{\sqrt{\left(\Upsilon-\alpha_{2}\right)\left(\alpha_{1}-\alpha_{3}\right)}+\sqrt{\left(\Upsilon-\alpha_{3}\right)\left(\alpha_{1}-\alpha_{2}\right)}}\right|, \\
& \quad \pm\left(\eta-\eta_{1}\right)=\frac{2 \alpha_{2}>\alpha_{3},}{\sqrt{\left(\alpha_{1}-\alpha_{3}\right)\left(\alpha_{2}-\alpha_{4}\right)}} F(\varphi, l), \\
& \alpha_{1}>\alpha_{2}>\alpha_{3}>\alpha_{4},
\end{aligned}
$$

where

$$
\begin{gathered}
A_{2}=\sqrt{-\frac{2 k^{2}\left(2+3 p+p^{2}\right)}{p^{2} \tau_{2}},} \\
\varphi=\arcsin \sqrt{\frac{\left(\Upsilon-\alpha_{1}\right)\left(\alpha_{2}-\alpha_{4}\right)}{\left(\Upsilon-\alpha_{2}\right)\left(\alpha_{1}-\alpha_{4}\right)}}, \\
l^{2}=\frac{\left(\alpha_{2}-\alpha_{3}\right)\left(\alpha_{1}-\alpha_{4}\right)}{\left(\alpha_{1}-\alpha_{3}\right)\left(\alpha_{2}-\alpha_{4}\right)} .
\end{gathered}
$$

Also $\alpha_{1}, \alpha_{2}, \alpha_{3}$, and $\alpha_{4}$ are the roots of the polynomial equation

$$
\Upsilon^{4}+\frac{\xi_{3}}{\xi_{4}} \Upsilon^{3}+\frac{\xi_{2}}{\xi_{4}} \Upsilon^{2}+\frac{\xi_{1}}{\xi_{4}} \Upsilon+\frac{\xi_{0}}{\xi_{4}}=0
$$

Substituting the solutions (42) into (8) and (15), we have

$$
\begin{aligned}
& u(x, t)=\left[\tau_{0}+\tau_{1} \alpha_{1} \pm \frac{\tau_{1} A_{2}}{k x+\left(k\left(3 \xi_{4} \tau_{1}^{2}-2 \xi_{2} \tau_{2}^{2}\right) / 2\left(2+3 p+p^{2}\right) \xi_{4} \tau_{2} \Gamma(1+\alpha)\right) t^{\alpha}-\eta_{0}}\right. \\
& \left.+\tau_{2}\left(\alpha_{1} \pm \frac{A_{2}}{k x+\left(k\left(3 \xi_{4} \tau_{1}^{2}-2 \xi_{2} \tau_{2}^{2}\right) / 2\left(2+3 p+p^{2}\right) \xi_{4} \tau_{2} \Gamma(1+\alpha)\right) t^{\alpha}-\eta_{0}}\right)^{2}\right]^{1 / p}, \\
& u(x, t)=\left\{\tau_{0}+\tau_{1} \alpha_{1}+\frac{4 A_{2}^{2}\left(\alpha_{2}-\alpha_{1}\right) \tau_{1}}{4 A_{2}^{2}-\left[\left(\alpha_{1}-\alpha_{2}\right)\left(k x+\left(k\left(3 \xi_{4} \tau_{1}^{2}-2 \xi_{2} \tau_{2}^{2}\right) / 2\left(2+3 p+p^{2}\right) \xi_{4} \tau_{2} \Gamma(1+\alpha)\right) t^{\alpha}-\eta_{0}\right)\right]^{2}}\right. \\
& \left.+\tau_{2}\left(\alpha_{1}+\frac{4 A_{2}^{2}\left(\alpha_{2}-\alpha_{1}\right)}{4 A_{2}^{2}-\left[\left(\alpha_{1}-\alpha_{2}\right)\left(k x+\left(k\left(3 \xi_{4} \tau_{1}^{2}-2 \xi_{2} \tau_{2}^{2}\right) / 2\left(2+3 p+p^{2}\right) \xi_{4} \tau_{2} \Gamma(1+\alpha)\right) t^{\alpha}-\eta_{0}\right)\right]^{2}}\right)^{2}\right\}^{1 / p}, \\
& u(x, t)=\left\{\tau_{0}+\tau_{1} \alpha_{2}+\frac{\left(\alpha_{2}-\alpha_{1}\right) \tau_{1}}{\exp \left[\left(\left(\alpha_{1}-\alpha_{2}\right) / A_{2}\right)\left(k x+\left(k\left(3 \xi_{4} \tau_{1}^{2}-2 \xi_{2} \tau_{2}^{2}\right) / 2\left(2+3 p+p^{2}\right) \xi_{4} \tau_{2} \Gamma(1+\alpha)\right) t^{\alpha}-\eta_{0}\right)\right]-1}\right. \\
& \left.+\tau_{2}\left(\alpha_{2}+\frac{\left(\alpha_{2}-\alpha_{1}\right)}{\exp \left[\left(\left(\alpha_{1}-\alpha_{2}\right) / A_{2}\right)\left(k x+\left(k\left(3 \xi_{4} \tau_{1}^{2}-2 \xi_{2} \tau_{2}^{2}\right) / 2\left(2+3 p+p^{2}\right) \xi_{4} \tau_{2} \Gamma(1+\alpha)\right) t^{\alpha}-\eta_{0}\right)\right]-1}\right)^{2}\right\}^{1 / p}, \\
& u(x, t)=\left\{\tau_{0}+\tau_{1} \alpha_{1}+\frac{\left(\alpha_{1}-\alpha_{2}\right) \tau_{1}}{\exp \left[\left(\left(\alpha_{1}-\alpha_{2}\right) / A_{2}\right)\left(k x+\left(k\left(3 \xi_{4} \tau_{1}^{2}-2 \xi_{2} \tau_{2}^{2}\right) / 2\left(2+3 p+p^{2}\right) \xi_{4} \tau_{2} \Gamma(1+\alpha)\right) t^{\alpha}-\eta_{0}\right)\right]-1}\right. \\
& \left.+\tau_{2}\left(\alpha_{1}+\frac{\left(\alpha_{1}-\alpha_{2}\right)}{\exp \left[\left(\left(\alpha_{1}-\alpha_{2}\right) / A_{2}\right)\left(k x+\left(k\left(3 \xi_{4} \tau_{1}^{2}-2 \xi_{2} \tau_{2}^{2}\right) / 2\left(2+3 p+p^{2}\right) \xi_{4} \tau_{2} \Gamma(1+\alpha)\right) t^{\alpha}-\eta_{0}\right)\right]-1}\right)^{2}\right\}^{1 / p},
\end{aligned}
$$




$$
\begin{aligned}
& u(x, t) \\
& =\left\{\tau_{0}+\tau_{1} \alpha_{1}-\left(\left(2\left(\alpha_{1}-\alpha_{2}\right)\left(\alpha_{1}-\alpha_{3}\right) \tau_{1}\right)\right.\right. \\
& \left.\times\left(2 \alpha_{1}-\alpha_{2}-\alpha_{3}+\left(\alpha_{3}-\alpha_{2}\right) \cosh \left[\frac{\sqrt{\left(\alpha_{1}-\alpha_{2}\right)\left(\alpha_{1}-\alpha_{3}\right)}}{A_{2}}\left(k x+\frac{k\left(3 \xi_{4} \tau_{1}^{2}-2 \xi_{2} \tau_{2}^{2}\right)}{2\left(2+3 p+p^{2}\right) \xi_{4} \tau_{2} \Gamma(1+\alpha)} t^{\alpha}-\eta_{0}\right)\right]\right)^{-1}\right) \\
& +\tau_{2}\left(\alpha_{1}-\left(\left(2\left(\alpha_{1}-\alpha_{2}\right)\left(\alpha_{1}-\alpha_{3}\right)\right)\right.\right. \\
& \left.\left.\left.\times\left(2 \alpha_{1}-\alpha_{2}-\alpha_{3}+\left(\alpha_{3}-\alpha_{2}\right) \cosh \left[\frac{\sqrt{\left(\alpha_{1}-\alpha_{2}\right)\left(\alpha_{1}-\alpha_{3}\right)}}{A_{2}}\left(k x+\frac{k\left(3 \xi_{4} \tau_{1}^{2}-2 \xi_{2} \tau_{2}^{2}\right)}{2\left(2+3 p+p^{2}\right) \xi_{4} \tau_{2} \Gamma(1+\alpha)} t^{\alpha}-\eta_{0}\right)\right]\right)^{-1}\right)\right)^{2}\right\}^{1 / p} \\
& u(x, t) \\
& =\left\{\tau_{0}+\tau_{1} \alpha_{2}+\left(\left(\tau_{1}\left(\alpha_{1}-\alpha_{2}\right)\left(\alpha_{4}-\alpha_{2}\right)\right)\right.\right. \\
& \times\left(\alpha_{4}-\alpha_{2}+\left(\alpha_{1}-\alpha_{4}\right) \operatorname{sn}^{2}\left[\frac{\sqrt{\left(\alpha_{1}-\alpha_{3}\right)\left(\alpha_{2}-\alpha_{4}\right)}}{2 A_{2}}\left(k x+\frac{k\left(3 \xi_{4} \tau_{1}^{2}-2 \xi_{2} \tau_{2}^{2}\right)}{2\left(2+3 p+p^{2}\right) \xi_{4} \tau_{2} \Gamma(1+\alpha)} t^{\alpha}-\eta_{0}\right)\right.\right. \\
& \left.\left.\left.\times \frac{\left(\alpha_{2}-\alpha_{3}\right)\left(\alpha_{1}-\alpha_{4}\right)}{\left(\alpha_{1}-\alpha_{3}\right)\left(\alpha_{2}-\alpha_{4}\right)}\right]\right)^{-1}\right) \\
& +\tau_{2}\left(\alpha_{2}+\left(\left(\tau_{1}\left(\alpha_{1}-\alpha_{2}\right)\left(\alpha_{4}-\alpha_{2}\right)\right)\right.\right. \\
& \times\left(\alpha_{4}-\alpha_{2}+\left(\alpha_{1}-\alpha_{4}\right) \operatorname{sn}^{2}\left[\frac{\sqrt{\left(\alpha_{1}-\alpha_{3}\right)\left(\alpha_{2}-\alpha_{4}\right)}}{2 A_{2}}\left(k x+\frac{k\left(3 \xi_{4} \tau_{1}^{2}-2 \xi_{2} \tau_{2}^{2}\right)}{2\left(2+3 p+p^{2}\right) \xi_{4} \tau_{2} \Gamma(1+\alpha)} t^{\alpha}-\eta_{0}\right),\right.\right. \\
& \left.\left.\left.\left.\left.\frac{\left(\alpha_{2}-\alpha_{3}\right)\left(\alpha_{1}-\alpha_{4}\right)}{\left(\alpha_{1}-\alpha_{3}\right)\left(\alpha_{2}-\alpha_{4}\right)}\right]\right)^{-1}\right)\right)^{2}\right\}^{1 / p} \text {. }
\end{aligned}
$$

For simplicity, if we take $\eta_{0}=0$, then we can write the solutions (45) as follows:

$$
u(x, t)=\left[\sum_{i=0}^{2} \tau_{i}\left(\alpha_{1} \pm \frac{A_{2}}{k\left(x-\lambda_{2} t^{\alpha}\right)}\right)^{i}\right]^{1 / p},
$$

$u(x, t)$

$$
\begin{aligned}
& =\left[\sum_{i=0}^{2} \tau_{i}\left(\alpha_{1}+\frac{4 A_{2}^{2}\left(\alpha_{2}-\alpha_{1}\right)}{4 A_{2}^{2}-\left[\left(\alpha_{1}-\alpha_{2}\right) k\left(x-\lambda_{2} t^{\alpha}\right)\right]^{2}}\right)^{i}\right]^{1 / p}, \\
& u(x, t)=\left[\sum_{i=0}^{2} \tau_{i}\left(\alpha_{2}+\frac{\alpha_{2}-\alpha_{1}}{\exp \left[B_{3}\left(x-\lambda_{2} t^{\alpha}\right)\right]-1}\right)^{i}\right]^{1 / p}, \\
& u(x, t)=\left[\sum_{i=0}^{2} \tau_{i}\left(\alpha_{1}+\frac{\alpha_{1}-\alpha_{2}}{\exp \left[B_{3}\left(x-\lambda_{2} t^{\alpha}\right)\right]-1}\right)^{i}\right]^{1 / p}
\end{aligned}
$$

$$
\begin{aligned}
& u(x, t) \\
& =\left[\sum _ { i = 0 } ^ { 2 } \tau _ { i } \left(\alpha_{1}-(\right.\right. \\
& \quad\left(2\left(\alpha_{1}-\alpha_{2}\right)\left(\alpha_{1}-\alpha_{3}\right)\right) \\
& \times\left(2 \alpha_{1}-\alpha_{2}-\alpha_{3}+\left(\alpha_{3}-\alpha_{2}\right)\right. \\
& \left.\left.\left.\left.\times \cosh \left[C\left(x-\lambda_{2} t^{\alpha}\right)\right]\right)^{-1}\right)\right)^{i}\right]^{1 / p},
\end{aligned}
$$

$u(x, t)$$$
=\left[\sum_{i=0}^{2} \tau_{i}\left(\alpha_{2}+\frac{\left(\alpha_{1}-\alpha_{2}\right)\left(\alpha_{4}-\alpha_{2}\right)}{\alpha_{4}-\alpha_{2}+\left(\alpha_{1}-\alpha_{4}\right) \operatorname{sn}^{2}(\varphi, l)}\right)^{i}\right]^{1 / p},
$$

where $B_{3}=k\left(\alpha_{1}-\alpha_{2}\right) / A_{2}, C=k \sqrt{\left(\alpha_{1}-\alpha_{2}\right)\left(\alpha_{1}-\alpha_{3}\right)} / A_{2}$, $\varphi=\left(k \sqrt{\left(\alpha_{1}-\alpha_{3}\right)\left(\alpha_{2}-\alpha_{4}\right)} / 2 A_{2}\right)\left(x-\lambda_{2} t^{\alpha}\right), l^{2}=\left(\alpha_{2}-\alpha_{3}\right)\left(\alpha_{1}-\right.$ $\left.\alpha_{4}\right) /\left(\alpha_{1}-\alpha_{3}\right)\left(\alpha_{2}-\alpha_{4}\right)$, and $\lambda_{2}=\left(3 \xi_{4} \tau_{1}^{2}-2 \xi_{2} \tau_{2}^{2}\right) / 2(2+3 p+$ $\left.p^{2}\right) \xi_{4} \tau_{2} \Gamma(1+\alpha)$. Here, $A_{2}$ is the amplitude of the soliton, while $\lambda_{2}$ is the velocity and $B_{3}$ and $C$ are the inverse width of the solitons. 
Remark 3. To our knowledge, the solutions (46) that we find in this paper have not been found in the literature, and these are new exact solutions of (1).

3.2. Application to the Fractional $K(n, n)$ Equation. In the case of $\alpha=1$, (2) reduces to the classical nonlinear generalized $K(n, n)$ equation. Many authors have studied to find the exact solutions of this equation by using a variety of methods $[27,28]$. In this paper, we consider the generalized fractional $K(n, n)$ equation for the case of $0<\alpha \leq 1$. Compactons, solitary patterns, and periodic and solitary traveling plane waves solutions of this equation are obtained.

Let us consider the travelling wave solutions of (2), and we perform the transformation $u(x, t)=u(\eta), \eta=k x-$ $\left(\lambda t^{\alpha} / \Gamma(1+\alpha)\right)$, where $\lambda$ is constant to be determined later. Then, integrating this equation with respect to $\eta$ and setting the integration constant to zero, we get

$$
k^{3}\left(u^{n}\right)^{\prime \prime}+k u^{n}-\lambda u=0 .
$$

By use of the following transformation:

$$
u=v^{1 /(n-1)},
$$

(47) turns into the equation

$$
k^{3}\left(n^{2}-n\right) v v^{\prime \prime}+k^{3} n\left(v^{\prime}\right)^{2}-\lambda(n-1)^{2} v+k(n-1)^{2} v^{2}=0 .
$$

From (8)-(10), we can write the following highest order nonlinear terms in order to determine the balance procedure:

$$
\begin{aligned}
v & =\tau_{\delta} \Upsilon^{\delta}+\cdots, \\
v^{2} & =\tau_{\delta}^{2} \Upsilon^{2 \delta}+\cdots, \\
v v^{\prime \prime} & =\omega \Upsilon^{2 \delta+\theta-\epsilon-2}+\cdots .
\end{aligned}
$$

Balancing the highest order nonlinear terms of $v v^{\prime \prime}$ and $v^{2}$ in (49), we have

$$
2 \delta+\theta-\epsilon-2=2 \delta,
$$

hence

$$
\theta=\epsilon+2
$$

This resolution procedure is applied and we obtain results as follows.

Case 1. If we take $\epsilon=0, \theta=2$, and $\delta=1$, then

$$
\left(v^{\prime}\right)^{2}=\frac{\tau_{1}^{2}\left(\xi_{2} \Upsilon^{2}+\xi_{1} \Upsilon+\xi_{0}\right)}{\zeta_{0}}, \quad v^{\prime \prime}=\frac{\tau_{1}\left(2 \xi_{2} \Upsilon+\xi_{1}\right)}{2 \zeta_{0}}
$$

where $\xi_{2} \neq 0, \zeta_{0} \neq 0$. Thus, we have a system of algebraic equations from the coefficients of polynomial of $u$. Solving the algebraic equation system (12), we get

$$
\begin{gathered}
\xi_{0}=\frac{\tau_{1}\left(\xi_{1} \tau_{1}-\xi_{2} \tau_{0}\right)}{\tau_{1}^{2}}, \quad \xi_{1}=\xi_{1}, \quad \xi_{2}=\xi_{2}, \\
\zeta_{0}=-\frac{k^{2} n^{2} \xi_{2}}{(n-1)^{2}}, \quad \tau_{0}=\tau_{0}, \quad \tau_{1}=\tau_{1}, \\
\lambda=\frac{k(n+1)\left(2 \xi_{2} \tau_{0}-\xi_{1} \tau_{1}\right)}{2 n \xi_{2}} .
\end{gathered}
$$

Substituting these results into (13), we have

$$
\begin{aligned}
& \pm\left(\eta-\eta_{0}\right) \\
& =\sqrt{-\frac{k^{2} n^{2}}{(n-1)^{2}}} \int \frac{d \Upsilon}{\sqrt{\Upsilon^{2}+\left(\xi_{1} / \xi_{2}\right) \Upsilon+\tau_{1}\left(\xi_{1} \tau_{1}-\xi_{2} \tau_{0}\right) / \tau_{1}^{2} \xi_{2}}}
\end{aligned}
$$

Integrating (55), we procure the solutions to (2) as follows:

$$
\begin{gathered}
\pm\left(\eta-\eta_{0}\right)=A_{3} \ln \left|\Upsilon-\alpha_{1}\right|, \\
\pm\left(\eta-\eta_{0}\right)=2 A_{3} \ln \left|\sqrt{\Upsilon-\alpha_{1}}+\sqrt{\Upsilon-\alpha_{2}}\right|,
\end{gathered}
$$

where $A_{3}=\sqrt{-\left(k^{2} n^{2} /(n-1)^{2}\right)}$. Also $\alpha_{1}, \alpha_{2}$ are the roots of the polynomial equation

$$
\Upsilon^{2}+\frac{\xi_{1}}{\xi_{2}} \Upsilon+\frac{\xi_{0}}{\xi_{2}}=0
$$

Substituting the solutions (56) into (8) and (48), we have

$$
\begin{gathered}
u(x, t)=\left[\tau_{0}+\tau_{1}\left(\exp \left[\frac{1}{A_{3}}\left(k x-\frac{k(n+1)\left(2 \xi_{2} \tau_{0}-\xi_{1} \tau_{1}\right) t^{\alpha}}{2 n \xi_{2} \Gamma(1+\alpha)}-\eta_{0}\right)\right]+\alpha_{1}\right)\right]^{1 /(n-1)}, \\
u(x, t)=\left[\tau_{0}+\tau_{1}\left(\left(\exp \left[\frac{1}{A_{3}}\left(k x-\frac{k(n+1)\left(2 \xi_{2} \tau_{0}-\xi_{1} \tau_{1}\right) t^{\alpha}}{2 n \xi_{2} \Gamma(1+\alpha)}-\eta_{0}\right)\right]\right.\right.\right. \\
\left.\left.\left.+\left(\alpha_{1}-\alpha_{2}\right)^{2} \exp \left[\frac{-1}{A_{3}}\left(k x-\frac{k(n+1)\left(2 \xi_{2} \tau_{0}-\xi_{1} \tau_{1}\right) t^{\alpha}}{2 n \xi_{2} \Gamma(1+\alpha)}-\eta_{0}\right)\right]+2\left(\alpha_{1}+\alpha_{2}\right)\right) \times(4)^{-1}\right)\right]^{1 /(n-1)} .
\end{gathered}
$$


If we take $\eta_{0}=\alpha_{2}=0$ and $\alpha_{1}=1$, then the solutions (58) can reduce to single kink solution and the hyperbolic function solution, respectively,

$$
\begin{gathered}
u(x, t)=\left(\tau_{0}+\tau_{1}\left(\exp \left[B_{4}\left(x-\lambda_{3} t^{\alpha}\right)\right]+1\right)\right)^{1 /(n-1)}, \\
u(x, t)=\left(\tau_{0}+\tau_{1}\left(\frac{1}{2}\left(\cosh \left[B_{4}\left(x-\lambda_{3} t^{\alpha}\right)\right]+1\right)\right)\right)^{1 /(n-1)},
\end{gathered}
$$

where $B_{4}=k / A_{3}, \lambda_{3}=(n+1)\left(2 \xi_{2} \tau_{0}-\xi_{1} \tau_{1}\right) / 2 n \xi_{2} \Gamma(1+\alpha)$. Here, $B_{4}$ is the inverse width of the solitons.

Remark 4. The solutions (59)-(60) obtained by using the extended trial equation method for (2) have been checked by Mathematica. To our knowledge, the hyperbolic function solution and single kink solution, that we find in this paper, are not shown in the previous literature. These results are new traveling wave solutions of (2).

Case 2. If we take $\epsilon=0, \theta=2$, and $\delta=2$, then

$$
\begin{gathered}
\left(v^{\prime}\right)^{2}=\frac{\left(\tau_{1}+2 \tau_{2} \Upsilon\right)^{2}\left(\xi_{2} \Upsilon^{2}+\xi_{1} \Upsilon+\xi_{0}\right)}{\zeta_{0}}, \\
v^{\prime \prime}=\frac{4 \tau_{2}\left(\xi_{2} \Upsilon^{2}+\xi_{1} \Upsilon+\xi_{0}\right)+\left(\tau_{1}+2 \tau_{1} \Upsilon\right)\left(2 \xi_{2} \Upsilon+\xi_{1}\right)}{2 \zeta_{0}},
\end{gathered}
$$

where $\xi_{2} \neq 0, \zeta_{0} \neq 0$. Thus, we have a system of algebraic equations from the coefficients of polynomial of $u$. Solving the algebraic equation system (12), we get

$$
\begin{gathered}
\xi_{0}=\xi_{0}, \quad \xi_{1}=\frac{\xi_{2} \tau_{1}}{\tau_{2}}, \quad \xi_{2}=\xi_{2}, \\
\zeta_{0}=-\frac{4 k^{2} n^{2} \xi_{2}}{(n-1)^{2}}, \quad \tau_{0}=\frac{\tau_{1}^{2}}{4 \tau_{2}}, \quad \tau_{1}=\tau_{1}, \\
\tau_{2}=\tau_{2}, \quad \lambda=\frac{k(n+1)\left(\xi_{2} \tau_{1}^{2}-4 \xi_{0} \tau_{2}^{2}\right)}{8 n \xi_{2} \tau_{2}} .
\end{gathered}
$$

Substituting these results into (13), we have

$$
\pm\left(\eta-\eta_{0}\right)=\sqrt{-\frac{4 k^{2} n^{2}}{(n-1)^{2}}} \int \frac{d \Upsilon}{\sqrt{\Upsilon^{2}+\left(\tau_{1} / \tau_{2}\right) \Upsilon+\left(\xi_{0} / \xi_{2}\right)}} .
$$

Integrating (63), we procure the solutions to (2) as follows:

$$
\begin{gathered}
\pm\left(\eta-\eta_{0}\right)=A_{4} \ln \left|\Upsilon-\alpha_{1}\right|, \\
\pm\left(\eta-\eta_{0}\right)=2 A_{4} \ln \left|\sqrt{\Upsilon-\alpha_{1}}+\sqrt{\Upsilon-\alpha_{2}}\right|,
\end{gathered}
$$

where $A_{4}=\sqrt{-\left(4 k^{2} n^{2} /(n-1)^{2}\right)}$. Also $\alpha_{1}, \alpha_{2}$ are the roots of the polynomial equation

$$
\Upsilon^{2}+\frac{\xi_{1}}{\xi_{2}} \Upsilon+\frac{\xi_{0}}{\xi_{2}}=0
$$

Substituting the solutions (64) into (8) and (48), we have

$$
\begin{aligned}
u(x, t)=\left\{\tau_{0}\right. & +\tau_{1}\left(\exp \left[\frac{1}{A_{4}}\left(k x-\frac{k(n+1)\left(\xi_{2} \tau_{1}^{2}-4 \xi_{0} \tau_{2}^{2}\right) t^{\alpha}}{8 n \xi_{2} \tau_{2} \Gamma(1+\alpha)}-\eta_{0}\right)\right]+\alpha_{1}\right) \\
& \left.+\tau_{2}\left(\exp \left[\frac{1}{A_{4}}\left(k x-\frac{k(n+1)\left(\xi_{2} \tau_{1}^{2}-4 \xi_{0} \tau_{2}^{2}\right) t^{\alpha}}{8 n \xi_{2} \tau_{2} \Gamma(1+\alpha)}-\eta_{0}\right)\right]+\alpha_{1}\right)^{2}\right\}^{1 /(n-1)},
\end{aligned}
$$

$u(x, t)$

$$
\begin{gathered}
=\left\{\tau_{0}+\tau_{1}\left(\left(\exp \left[\frac{1}{A_{4}}\left(k x-\frac{k(n+1)\left(\xi_{2} \tau_{1}^{2}-4 \xi_{0} \tau_{2}^{2}\right) t^{\alpha}}{8 n \xi_{2} \tau_{2} \Gamma(1+\alpha)}-\eta_{0}\right)\right]\right.\right.\right. \\
\left.\left.+\left(\alpha_{1}-\alpha_{2}\right)^{2} \exp \left[\frac{-1}{A_{4}}\left(k x-\frac{k(n+1)\left(\xi_{2} \tau_{1}^{2}-4 \xi_{0} \tau_{2}^{2}\right) t^{\alpha}}{8 n \xi_{2} \tau_{2} \Gamma(1+\alpha)}-\eta_{0}\right)\right]+2\left(\alpha_{1}+\alpha_{2}\right)\right) \times(4)^{-1}\right) \\
+\tau_{2}\left(\left(\exp \left[\frac{1}{A_{4}}\left(k x-\frac{k(n+1)\left(\xi_{2} \tau_{1}^{2}-4 \xi_{0} \tau_{2}^{2}\right) t^{\alpha}}{8 n \xi_{2} \tau_{2} \Gamma(1+\alpha)}-\eta_{0}\right)\right]\right.\right. \\
\left.\left.\left.+\left(\alpha_{1}-\alpha_{2}\right)^{2} \exp \left[\frac{-1}{A_{4}}\left(k x-\frac{k(n+1)\left(\xi_{2} \tau_{1}^{2}-4 \xi_{0} \tau_{2}^{2}\right) t^{\alpha}}{8 n \xi_{2} \tau_{2} \Gamma(1+\alpha)}-\eta_{0}\right)\right]+2\left(\alpha_{1}+\alpha_{2}\right)\right) \times(4)^{-1}\right)^{2}\right\}^{1 /(n-1)} .
\end{gathered}
$$


For simplicity, $\eta_{0}=\alpha_{2}=0$ and $\alpha_{1}=1$, then we can write the solutions (66) as follows:

$$
\begin{gathered}
u(x, t)=\left[\sum_{i=0}^{2} \tau_{i}\left(\exp \left[B_{5}\left(x-\lambda_{4} t^{\alpha}\right)+1\right]\right)^{i}\right]^{1 /(n-1)}, \\
u(x, t)=\left[\sum_{i=0}^{2} \tau_{i}\left(\frac{1}{2}\left(\cosh \left[B_{5}\left(x-\lambda_{4} t^{\alpha}\right)\right]+1\right)\right)^{i}\right]^{1 /(n-1)},
\end{gathered}
$$

where $B_{5}=k / A_{4}, \lambda_{4}=(n+1)\left(\xi_{2} \tau_{1}^{2}-4 \xi_{0} \tau_{2}^{2}\right) / 8 n \xi_{2} \tau_{2} \Gamma(1+\alpha)$. Here, $B_{5}$ is the inverse width of the solitons.

Case 3. If we take $\epsilon=1, \theta=3$, and $\delta=1$, then

$$
\begin{aligned}
&\left(v^{\prime}\right)^{2}= \frac{\tau_{1}^{2}\left(\xi_{3} \Upsilon^{3}+\xi_{2} \Upsilon^{2}+\xi_{1} \Upsilon+\xi_{0}\right)}{\zeta_{0}+\zeta_{1} \Upsilon}, \\
& v^{\prime \prime}=\left(\tau _ { 1 } \left(\left(\zeta_{0}+\zeta_{1} \Upsilon\right)\left(3 \xi_{3} \Upsilon^{2}+2 \xi_{2} \Upsilon+\xi_{1}\right)\right.\right. \\
&\left.\left.\quad-\zeta_{1}\left(\xi_{3} \Upsilon^{3}+\xi_{2} \Upsilon^{2}+\xi_{1} \Upsilon+\xi_{0}\right)\right)\right) \\
& \times\left(2\left(\zeta_{0}+\zeta_{1} \Upsilon\right)^{2}\right)^{-1},
\end{aligned}
$$

where $\xi_{3} \neq 0, \zeta_{1} \neq 0$. Respectively, solving the algebraic equation system (12) yields

$$
\begin{aligned}
& \xi_{0}=-\frac{(n-1)^{2} \zeta_{0} \tau_{0}\left((n-1)^{2} \zeta_{0} \tau_{0}+k^{2} n^{2} \xi_{1} \tau_{1}\right)}{k^{2} n^{2} \tau_{1}\left(k^{2} n^{2} \xi_{3} \tau_{0}-(n-1)^{2} \zeta_{0} \tau_{1}\right)}, \\
& \xi_{1}=\xi_{1} \\
& \xi_{2}=\left(k^{4} n^{4} \xi_{3}^{2} \tau_{0}^{2}+(n-1)^{4} \zeta_{0}^{2} \tau_{1}^{2}\right. \\
& \left.+k^{2} \xi_{3} \tau_{1}\left(k^{2} n^{4} \xi_{1} \tau_{1}-(n-1)^{2} n^{2} \zeta_{0} \tau_{0}\right)\right) \\
& \times\left(k^{2} \tau_{1}\left(k^{2} n^{4} \xi_{3} \tau_{0}-(n-1)^{2} n^{2} \zeta_{0} \tau_{1}\right)\right)^{-1}, \\
& \xi_{3}=\xi_{3}, \quad \zeta_{0}=\zeta_{0}, \quad \zeta_{1}=-\frac{k^{2} n^{2} \xi_{3}}{(n-1)^{2}}, \\
& \tau_{0}=\tau_{0}, \quad \tau_{1}=\tau_{1}, \\
& \lambda=\frac{k(n+1)\left(k^{2} n^{2} \xi_{3} \tau_{0}^{2}-\tau_{1}\left(2(n-1)^{2} \zeta_{0} \tau_{0}+k^{2} n^{2} \xi_{1} \tau_{1}\right)\right)}{2 k^{2} n^{3} \xi_{3} \tau_{0}-2(n-1)^{2} n \zeta_{0} \tau_{1}} .
\end{aligned}
$$

Substituting these results into (13), we have

$$
\begin{aligned}
\pm & \left(\eta-\eta_{0}\right) \\
& =\sqrt{-\frac{k^{2} n^{2}}{(n-1)^{2}}} \\
& \times \int \sqrt{\frac{\Upsilon+\left(\zeta_{0} / \zeta_{1}\right)}{\Upsilon^{3}+\left(\xi_{2} / \xi_{3}\right) \Upsilon^{2}+\left(\xi_{1} / \xi_{3}\right) \Upsilon+\left(\xi_{0} / \xi_{3}\right)}} d \Upsilon .
\end{aligned}
$$

Integrating (70), we obtain the solutions to (2) as follows:

$$
\begin{aligned}
\pm & \left(\eta-\eta_{0}\right) \\
= & 2 A_{3}\left(\ln \left|\sqrt{\frac{\zeta_{0}+\zeta_{1} \Upsilon}{\zeta_{1}}}+\sqrt{\Upsilon-\alpha_{1}}\right|-\sqrt{\frac{\zeta_{0}+\zeta_{1} \Upsilon}{\zeta_{1}\left(\Upsilon-\alpha_{1}\right)}}\right) \\
\pm & \left(\eta-\eta_{0}\right) \\
= & \frac{2 A_{3}}{\sqrt{\zeta_{1}\left(\alpha_{2}-\alpha_{1}\right)}} \\
& \times\left(\sqrt{\zeta_{0}+\zeta_{1} \alpha_{1}} \arctan \left[\sqrt{\frac{\left(\zeta_{0}+\zeta_{1} \alpha_{1}\right)\left(\Upsilon-\alpha_{2}\right)}{\left(\zeta_{0}+\zeta_{1} \Upsilon\right)\left(\alpha_{2}-\alpha_{1}\right)}}\right]\right. \\
\pm & \left(\eta-\eta_{0}\right) \\
= & \frac{\sqrt{\left(\alpha_{1}-\alpha_{2}\right) \zeta_{1}\left(\zeta_{0}+\zeta_{1} \alpha_{3}\right)}}{\left.\sqrt{\alpha_{2}}\left|\sqrt{\frac{\zeta_{0}+\zeta_{1} \Upsilon}{\zeta_{1}}}+\sqrt{\Upsilon-\alpha_{2}}\right|\right)} \\
& \times\left(\left(\zeta_{0}+\zeta_{1} \alpha_{1}\right) F(\varphi, l)+\left(\alpha_{3}-\zeta_{1} \alpha_{1}\right) \pi(\varphi, n, l)\right)
\end{aligned}
$$

where

$$
\begin{gathered}
F(\varphi, l)=\int_{0}^{\varphi} \frac{d \psi}{\sqrt{1-l^{2} \sin ^{2} \psi}}, \\
\pi(\varphi, n, l)=\int_{0}^{\varphi} \frac{d \psi}{\left(1+n \sin ^{2} \psi\right) \sqrt{1-l^{2} \sin ^{2} \psi}}, \\
\varphi=\arcsin \sqrt{\frac{\left(\Upsilon-\alpha_{3}\right)\left(\alpha_{2}-\alpha_{1}\right)}{\left(\Upsilon-\alpha_{1}\right)\left(\alpha_{2}-\alpha_{3}\right)}}, \\
l^{2}=\frac{\left(\zeta_{0}+\zeta_{1} \alpha_{1}\right)\left(\alpha_{3}-\alpha_{2}\right)}{\left(\zeta_{0}+\zeta_{1} \alpha_{3}\right)\left(\alpha_{1}-\alpha_{2}\right)}, \quad n=\frac{\alpha_{3}-\alpha_{2}}{\alpha_{1}-\alpha_{2}} .
\end{gathered}
$$

Also $\alpha_{1}, \alpha_{2}$, and $\alpha_{3}$ are the roots of the polynomial equation

$$
\Upsilon^{3}+\frac{a_{2}}{a_{3}} \Upsilon^{2}+\frac{a_{1}}{a_{3}} \Upsilon+\frac{a_{0}}{a_{3}}=0
$$

\section{Conclusions}

In this study, the extended trial equation method is applied successfully for solving the nonlinear fractional differential equations. We used it to obtain some soliton and elliptic function or elliptic integral function solutions, which are one-wave solutions, to the generalized third order fractional $\mathrm{KdV}$ equation and the fractional $K(n, n)$ equation. The performance of this method is reliable and effective, and also this method gives more general solutions. We think that the proposed method can also be applied to other fractional nonlinear differential equations with nonlinear evolution. In 
our future studies, we will define the multiple trial equation method for the fractional or nonfractional nonlinear partial differential equations.

\section{Appendix}

In [29], the first kind of the Legendre elliptic integral is given by

$$
\begin{aligned}
g(t) & =\int_{0}^{\vartheta} \frac{1}{\sqrt{1-m^{2} \sin ^{2} \hbar}} d \hbar \\
& =\int_{0}^{t \equiv \sin \hbar} \frac{1}{\sqrt{\left(1-x^{2}\right)\left(1-m^{2} x^{2}\right)}} d x,
\end{aligned}
$$

where $m$ is known as the modulus. Otherwise, the inverse function $t \equiv \sin \hbar$ is called as the Jacobi elliptic sine function. This function can be represented by $t=\operatorname{sn} u$.

When $m \rightarrow 0$, the Jacobi elliptic function sn $u$ is transformed into $\sin u$. If we take $m \rightarrow 1$, sn $u$ is degenerated as $\tanh u$.

\section{Acknowledgments}

The research has been supported by Yozgat University Foundation.

\section{References}

[1] J. A. T. Machado, M. F. Silva, R. S. Barbosa et al., "Some applications of fractional calculus in engineering," Mathematical Problems in Engineering, vol. 2010, Article ID 639801, 34 pages, 2010.

[2] M. Dalir and M. Bashour, "Applications of fractional calculus," Applied Mathematical Sciences, vol. 4, no. 21-24, pp. 1021-1032, 2010.

[3] K. S. Miller and B. Ross, An Introduction to the Fractional Calculus and Fractional Differential Equations, A Wiley-Interscience Publication, John Wiley \& Sons, New York, NY, USA, 1993.

[4] A. A. Kilbas, H. M. Srivastava, and J. J. Trujillo, Theory and Applications of Fractional Differential Equations, vol. 204 of North-Holland Mathematics Studies, Elsevier Science B.V., Amsterdam, The Netherlands, 2006.

[5] I. Podlubny, Fractional Differential Equations, vol. 198 of Mathematics in Science and Engineering, Academic Press, San Diego, Calif, USA, 1999.

[6] B. Lu, "The first integral method for some time fractional differential equations," Journal of Mathematical Analysis and Applications, vol. 395, no. 2, pp. 684-693, 2012.

[7] A. El-Kahlout, T. O. Salim, and S. El-Azab, "Exact solution of time fractional partial differential equation," Applied Mathematical Sciences, vol. 2, no. 49-52, pp. 2577-2590, 2008.

[8] R. Sahadevan and T. Bakkyaraj, "Invariant analysis of time fractional generalized Burgers and Korteweg-de Vries equations," Journal of Mathematical Analysis and Applications, vol. 393, no. 2, pp. 341-347, 2012.

[9] M. Dehghan, J. Manafian, and A. Saadatmandi, "Solving nonlinear fractional partial differential equations using the homotopy analysis method," Numerical Methods for Partial Differential Equations, vol. 26, no. 2, pp. 448-479, 2010.
[10] G. Jumarie, "Modified Riemann-Liouville derivative and fractional Taylor series of nondifferentiable functions further results," Computers \& Mathematics with Applications, vol. 51, no. 9-10, pp. 1367-1376, 2006.

[11] G. Jumarie, "Fractional Hamilton-Jacobi equation for the optimal control of nonrandom fractional dynamics with fractional cost function," Journal of Applied Mathematics \& Computing, vol. 23 , no. 1-2, pp. 215-228, 2007.

[12] G. Jumarie, "Table of some basic fractional calculus formulae derived from a modified Riemann-Liouville derivative for nondifferentiable functions," Applied Mathematics Letters, vol. 22, no. 3, pp. 378-385, 2009.

[13] Z. Z. Ganji, D. D. Ganji, A. D. Ganji, and M. Rostamian, "Analytical solution of time-fractional Navier-Stokes equation in polar coordinate by homotopy perturbation method," Numerical Methods for Partial Differential Equations, vol. 26, no. 1, pp. 117-124, 2010.

[14] C.-S. Liu, "Trial equation method and its applications to nonlinear evolution equations," Acta Physica Sinica, vol. 54, no. 6, pp. 2505-2509, 2005.

[15] C.-S. Liu, "Trial equation method for nonlinear evolution equations with rank inhomogeneous: mathematical discussions and applications," Communications in Theoretical Physics, vol. 45, pp. 219-223, 2006.

[16] C.-S. Liu, "A new trial equation method and its applications," Communications in Theoretical Physics, vol. 45, pp. 395-397, 2006.

[17] C.-S. Liu, "Applications of complete discrimination system for polynomial for classifications of traveling wave solutions to nonlinear differential equations," Computer Physics Communications, vol. 181, no. 2, pp. 317-324, 2010.

[18] Y.-J. Cheng, "Classification of traveling wave solutions to the Vakhnenko equations," Computers \& Mathematics with Applications, vol. 62, no. 10, pp. 3987-3996, 2011.

[19] Y.-J. Cheng, "Classification of traveling wave solutions to the modified form of the Degasperis-Procesi equation," Mathematical and Computer Modelling, vol. 56, no. 1-2, pp. 43-48, 2012.

[20] Y. Gurefe, A. Sonmezoglu, and E. Misirli, "Application of the trial equation method for solving some nonlinear evolution equations arising in mathematical physics," Pramana, vol. 77, no. 6, pp. 1023-1029, 2011.

[21] Y. Gurefe, A. Sonmezoglu, and E. Misirli, "Application of an irrational trial equation method to high-dimensional nonlinear evolution equations," Journal of Advanced Mathematical Studies, vol. 5, no. 1, pp. 41-47, 2012.

[22] Y. Pandir, Y. Gurefe, U. Kadak, and E. Misirli, "Classification of exact solutions for some nonlinear partial differential equations with generalized evolution," Abstract and Applied Analysis, vol. 2012, Article ID 478531, 16 pages, 2012.

[23] Y. Gurefe, E. Misirli, A. Sonmezoglu, and M. Ekici, "Extended trial equation method to generalized nonlinear partial differential equations," Applied Mathematics and Computation, vol. 219, no. 10, pp. 5253-5260, 2013.

[24] Y. Pandir, Y. Gurefe, and E. Misirli, "Classification of exact solutions to the generalized Kadomtsev-Petviashvili equation," Physica Scripta, vol. 87, Article ID 025003, 12 pages, 2013.

[25] M. V. Demina and N. A. Kudryashov, "From Laurent series to exact meromorphic solutions: the Kawahara equation," Physics Letters A, vol. 374, no. 39, pp. 4023-4029, 2010.

[26] N. A. Kudryashov and M. V. Demina, "Traveling wave solutions of the generalized nonlinear evolution equations," Applied Mathematics and Computation, vol. 210, no. 2, pp. 551-557, 2009. 
[27] A.-M. Wazwaz, "The variational iteration method for rational solutions for $\mathrm{KdV}, K(2,2)$, Burgers, and cubic Boussinesq equations," Journal of Computational and Applied Mathematics, vol. 207, no. 1, pp. 18-23, 2007.

[28] P. Rosenau and J. M. Hyman, "Compactons: solitons with finite wavelengths," Physical Review Letters, vol. 70, pp. 564-567, 1993.

[29] Z. Fu, S. Liu, S. Liu, and Q. Zhao, "New Jacobi elliptic function expansion and new periodic solutions of nonlinear wave equations," Physics Letters A, vol. 290, no. 1-2, pp. 72-76, 2001. 


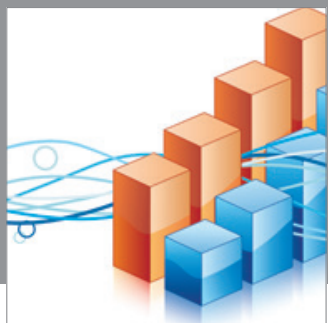

Advances in

Operations Research

mansans

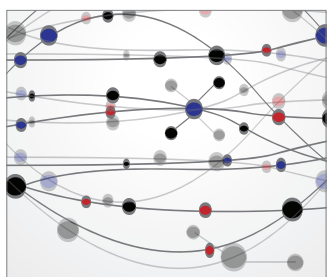

The Scientific World Journal
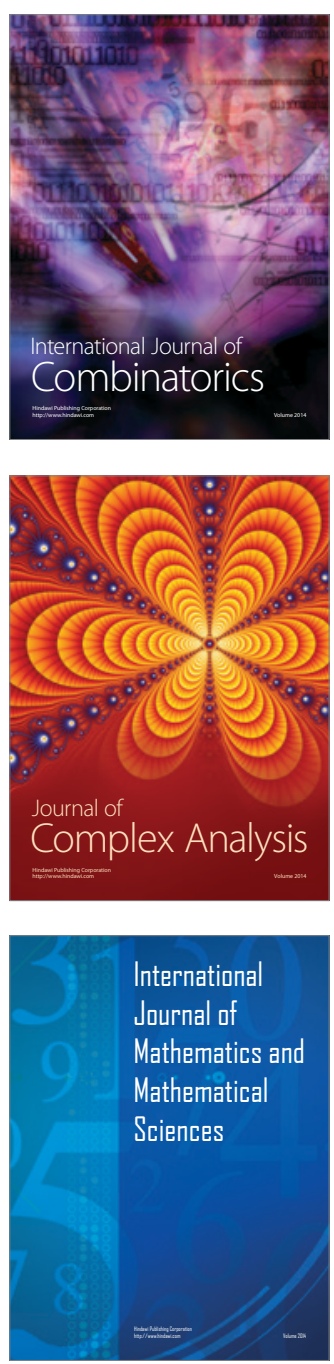
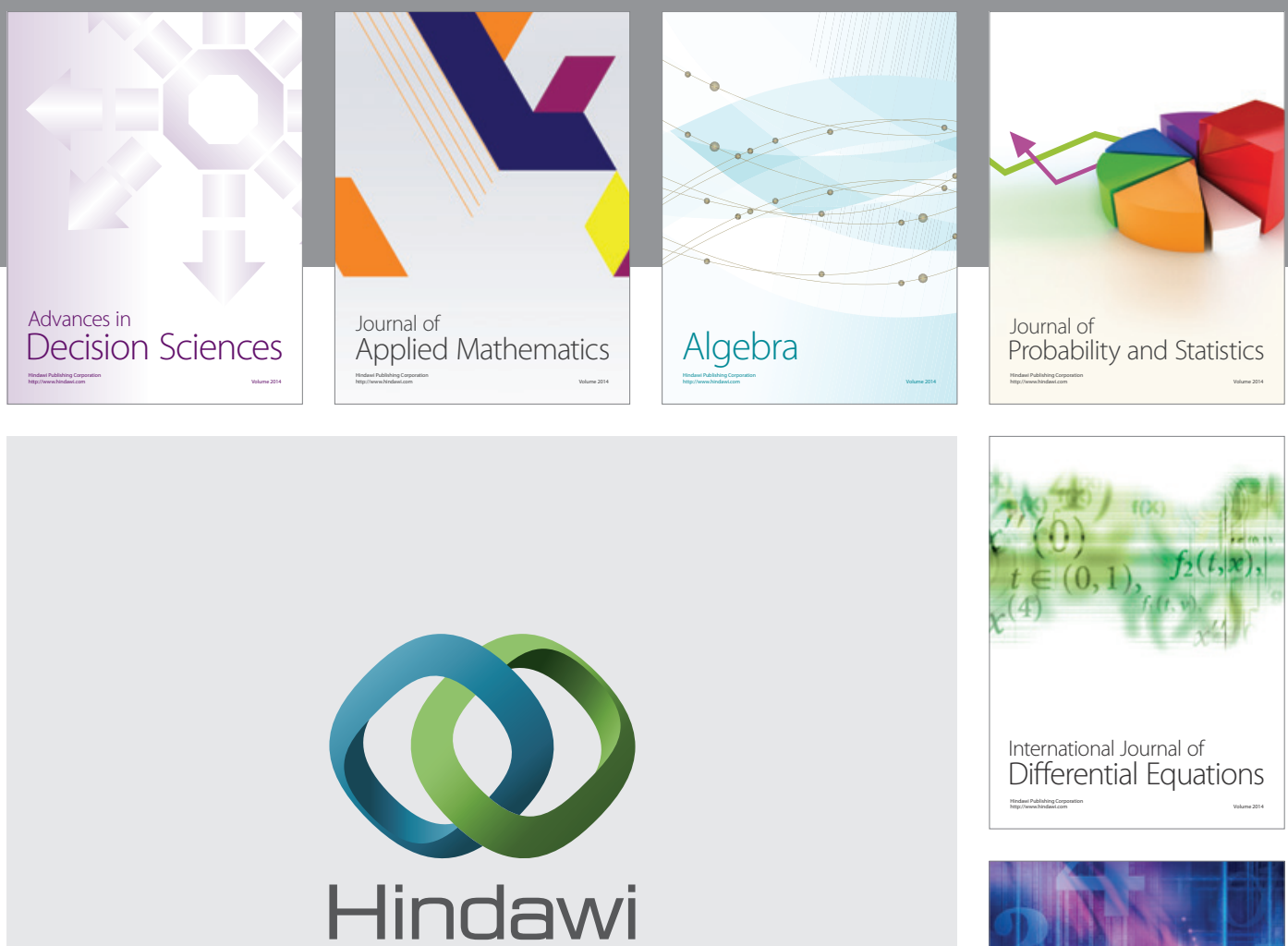

Submit your manuscripts at http://www.hindawi.com
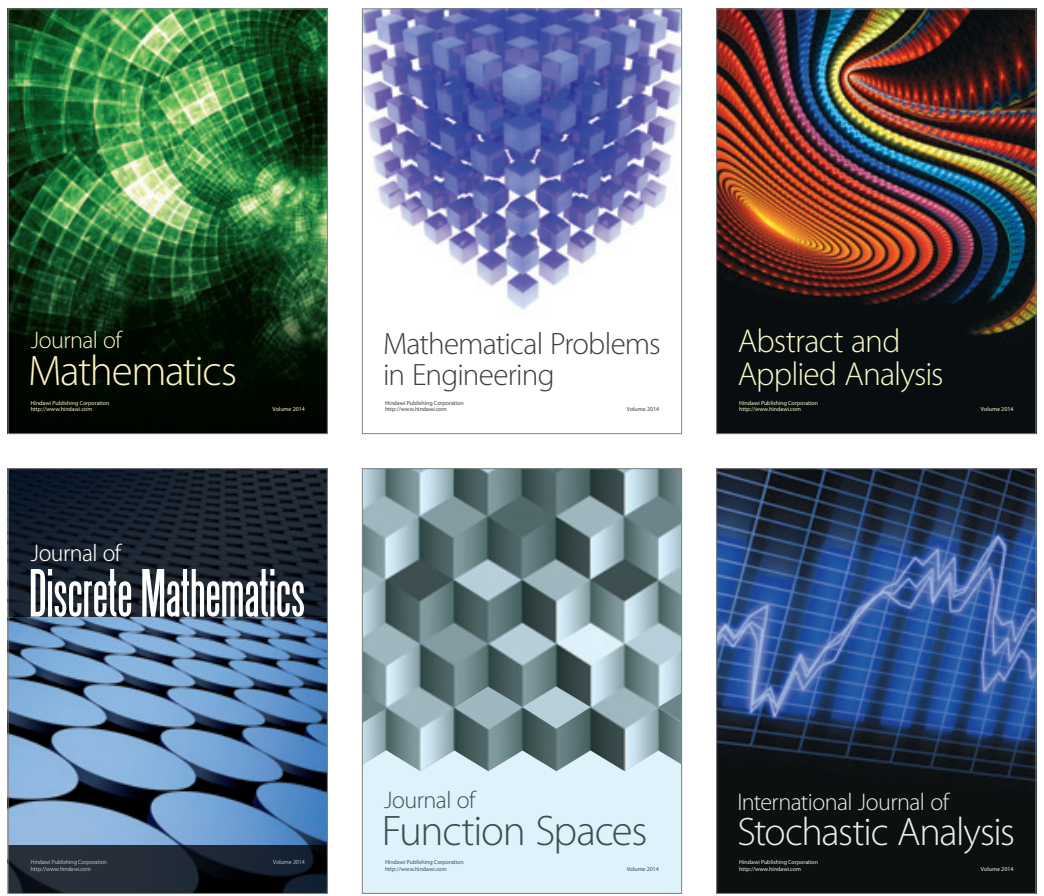

Journal of

Function Spaces

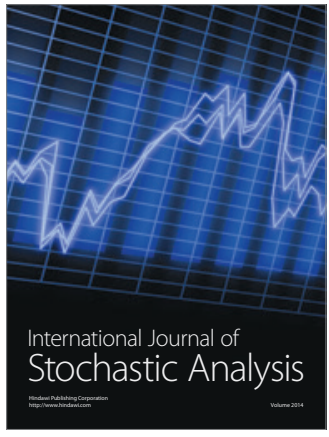

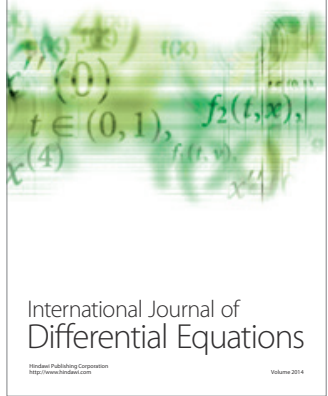
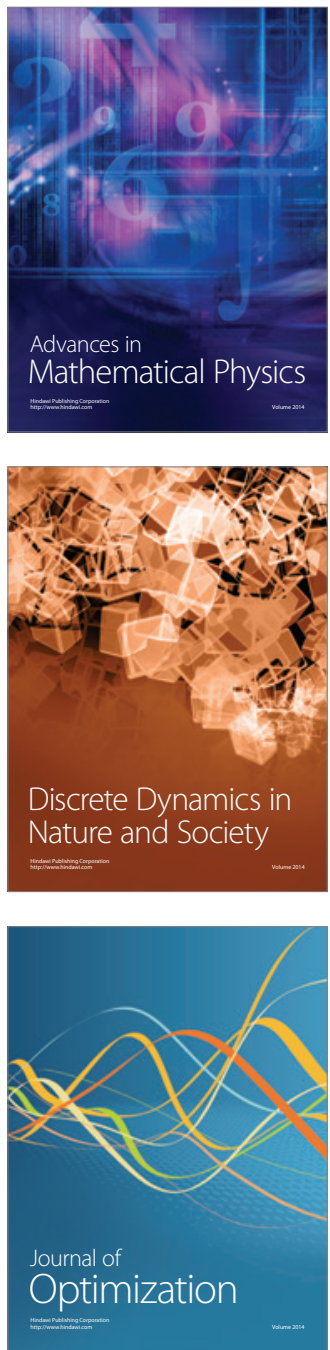